\title{
Programas de salud y alimentación escolar, el papel de la participación de la comunidad
}

\author{
School Health and Nutrition Programs, the Role of Community Participation
}

\author{
Erik Salazar-Pérez " , Marcos Galván $M M^{2}$, Guadalupe López-Rodríguez ${ }^{3}$, Jhazmín Hernández. \\ Cabrera ${ }^{4}$
}

\begin{abstract}
:
The aim of this paper was to identify the health and nutrition determinants from schoolchildren in the school context and analyzed the participation of the community to achieve the integral formation of schoolchildren. Health and nutrition programs are addressed, which are aimed at providing health education, physical education, health services, nutrition and food services to improve the health of schoolchildren, both physically and mentally and socially, which promote behaviors and healthy lifestyles. Included norms that regulate the participation of the community in school health and nutrition, and those that specifically address aspects of the life of the school to generate an atmosphere of safety, well-being and quality to ensure a better school and human development. To finally, analyzed the participation of the community in the school's health and nutrition; the parents or guardians are important in school and in the classroom, because they are the indirect recipients of the service offered, since without their collaboration and support it is more difficult to achieve the expected learning in the students. In conclusion, the school and the community are interrelated in a natural way, because the school is part of the community life, being necessary to improve the regulatory frameworks to ensure a true participation in the programs and actions of prevention and improvement of child health and nutrition, achieve the educational purposes and the integral formation of schoolchildren.
\end{abstract}

Keywords:

School children, community participation, school environment, health programs, nutrition programs

\section{Resumen:}

El objetivo de este trabajo fue identificar los determinantes de la salud y nutrición de los escolares en el contexto escolar y analizar la participación de la comunidad para lograr la formación integral de sus estudiantes. Se abordan los programas de salud y alimentación que van encaminados a brindar educación en salud, educación física, servicios de salud, servicios de nutrición y alimentación para mejorar la salud de los escolares, tanto en el aspecto físico como mental y social, que promueven comportamientos y estilos de vida saludables. Se incluyen normas que regulan la participación de la comunidad en la salud y nutrición escolar, y las que atienden de manera específica los aspectos de la vida del escolar para generan una atmósfera de seguridad, bienestar y calidad que garanticen un mejor desarrollo escolar y humano. Para finalmente, analizar la participación de la comunidad en la salud y nutrición del escolar; identificando que los padres de familia o tutores son importantes en la escuela y en el aula, porque son los destinatarios indirectos del servicio que se ofrece, ya que sin su colaboración y apoyo es más difícil lograr los aprendizajes esperados en los alumnos. Se concluye que la escuela y la comunidad están interrelacionadas de manera natural, porque la escuela forma parte de la vida comunitaria, siendo necesario mejorar los marcos normativos para asegurar una verdadera participación en los programas y acciones de prevención y mejoramiento de la salud y nutrición infantil, lograr los propósitos educativos y la formación integral del estudiante.

\footnotetext{
${ }^{1}$ Universidad Autónoma del Estado de Hidalgo, Licenciatura en Nutrición, Instituto de Ciencias de la Salud (ICSa), San Agustín Tlaxiaca, Hidalgo, México. ORCID: https://orcid.org/0000-0001-6794-803X, Email: salazarperezerik@ gmail.com

2 Autor de correspondencia. Universidad Autónoma del Estado de Hidalgo, Cuerpo Académico de Epidemiología Nutricional y Molecular, Instituto de Ciencias de la Salud, Hidalgo, México. ORCID: https://orcid.org/0000-0002-3254-4470, Email: mgalvan73@ hotmail.com

${ }^{3}$ Universidad Autónoma del Estado de Hidalgo, Cuerpo Académico de Epidemiología Nutricional y Molecular, Instituto de Ciencias de la Salud, Hidalgo, México. ORCID: https://orcid.org/0000-0001-5432-0382, Email: glopez.dra@ gmail.com

${ }^{4}$ Universidad Autónoma del Estado de Hidalgo, Área Académica de Nutrición, Instituto de Ciencias de la Salud, Hidalgo, México. ORCID: https://orcid.org/0000-0003-4712-4660,Email: jhazda@yahoo.com.mx
} 
Palabras Clave:

Escolares, participación comunitaria, ambiente escolar, programas de salud, programas de nutrición

\section{Determinantes de la salud y nutrición del escolar en las escuelas}

Aun cuando los países realizan esfuerzos importantes por mejorar la salud y nutrición de su infancia, generalmente estos esfuerzos se han realizado de forma aislada, sin considerar un marco general de los determinantes sociales de la salud y nutrición de los niños y niñas en el contexto escolar. Sabemos que cada individuo está expuesto a diferentes condiciones socioeconómicas, culturales y ambientales a nivel familiar y comunitario; sin embargo, poco se ha discutido sobre los determinantes de la salud y nutrición en el contexto escolar, en este trabajo se ha identificado como un primer determinante de la salud y nutrición a los programas y servicios con que cuenta la escuela, tanto en la atención directa de la salud y nutrición, como en las acciones de educación para la salud que realiza. Un segundo determinante, está constituido por el entorno psicosocial, la infraestructura física, seguridad pública, la disponibilidad de alimentos, y el transporte escolar. Identificando como un tercer determinante a las normas de organización de la escuela, y finalmente el cuarto determinante, esta constituido por la participación de la comunidad. Estos determinantes son abordados a lo largo de este trabajo, sin pretender ser una revisión exhaustiva, y será necesario realizar en una segunda entrega las recomendaciones pertinentes basadas en evidencia científica, que sirvan como insumo para mejorar las intervenciones y políticas dirigidas a mejorar la salud y disminuir las inequidades en los niños y niñas de México (Figura 1).

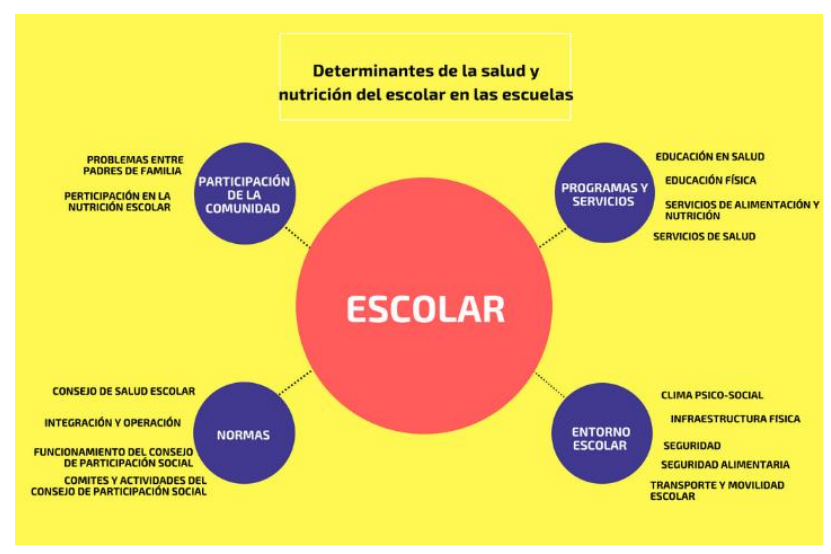

Figura.1- Determinantes de la salud y nutrición del escolar en las escuelas.

\section{1.- Programas y servicios proporcionados a los estudiantes en las escuelas}

Actualmente los servicios que son proporcionados a la comunidad escolar en las escuelas mexicanas van encaminados a brindar educación en salud, educación física, alimentación escolar y salud, lo cual tiene por objetivo mejorar la salud de los escolares, tanto a nivel físico como a nivel mental y social, promoviendo comportamientos y estilos de vida saludables.

\section{1- Educación en salud}

La educación para la salud como disciplina surge ante la necesidad de proporcionar a las personas los medios necesarios para mejorar la salud, a través de la modificación de conductas de riesgo. En la 36 Asamblea Mundial de la Salud se definió a la educación para la salud como cualquier combinación de actividades de información y educación que lleve a una situación en la que las personas sepan cómo alcanzar niveles de salud óptimos y busquen ayuda cuando lo necesiten. La educación para la salud tiene como objetivo primordial diseñar programas de intervención destinados a modificar creencias, costumbres y hábitos no saludables, además de promover, proteger y fomentar la salud. En el aspecto nutricional, la educación para la salud como estrategia en las escuelas está dirigida a fomentar hábitos alimentarios saludables que contrarresten la prevalencia de mala nutrición (desnutrición y obesidad) a través de la promoción de hábitos alimentarios y estilos de vida saludables en los escolares, que inculque en los niños actitudes, conocimientos y hábitos saludables que favorezcan su crecimiento y desarrollo, y el fomento de su salud y la prevención de las enfermedades evitables de su edad. ${ }^{1}$ En el aula de educación a nivel primaria, los estudiantes aprenden el conocimiento esencial y las habilidades que pueden emplear para participar en comportamientos saludables. La instrucción sobre la salud se puede proporcionar a través de otros componentes del programa de salud escolar, incluida la educación física, el asesoramiento escolar y los servicios de salud escolar, y puede integrarse en otras asignaturas, como las matemáticas, ciencias naturales o sociales. ${ }^{2}$

La educación para la salud forma parte indiscutible del proceso educativo cuya función es formar de manera integral a los alumnos. Se trata tanto de transmitir conocimientos como de favorecer la adquisición de habilidades que ayuden a afrontar la realidad del modo más eficaz y, para lograr este objetivo, se requiere de la participación y el compromiso de todos.

\section{2- Educación Física}


En la asignatura de Educación Física, se encuentran presentes aprendizajes actitudinales, para lograr incidir en los hábitos de vida activa y saludable de los escolares. ${ }^{3}$ La actividad física como hábito, es una herramienta que ha mostrado su efecto en la prevención de enfermedades crónicas no transmisibles las cuales son la primera causa de mortalidad en nuestro país. La actividad física se define como cualquier movimiento corporal producido por los músculos esqueléticos y que requiere cierto gasto energético, por otro lado, la inactividad física se relaciona con actividades sedentarias y es una variable asociada a la obesidad, generando un desequilibrio entre la ingesta y el gasto energético. Se sabe que la actividad física practicada regularmente a una intensidad moderada y con una frecuencia específica, provee beneficios a la salud mejorando la capacidad cardiorrespiratoria, tensión arterial, fortalecimiento de huesos y músculos, reduce los niveles de colesterol de baja densidad (LDL), aumenta el colesterol de alta densidad (HDL) y ayuda a mantener una adecuada composición corporal; así como a regular los niveles de estrés y depresión. ${ }^{4}$

La actividad física es uno de los principales factores que condicionan la salud humana, de tal modo que la inactividad física o sedentarismo constituye el cuarto factor de riesgo más importante de mortalidad en todo el mundo. En los niños sus beneficios actúan sobre los procesos de crecimiento y desarrollo, y la prevención de factores de riesgo de enfermedades cardiovasculares, e incluso en el mejoramiento de su desempeño académico. $^{5}$

\section{3- Servicios de alimentación y nutrición}

Los escolares que tienen acceso a alimentos saludables en la escuela suelen transmitir la información entre los miembros de su familia, y van generando prácticas saludables desde su educación primaria, por lo que puede ser potencialmente más sano en la edad adulta. Sin embargo, el consumo de alimentos con ingredientes que no favorecen a la salud se ha incrementado en los últimos años, tales como grasas saturadas, azúcar, sodio y otros. Si bien la elección y consumo de estos alimentos se debe en gran parte a las decisiones individuales; existe una fuerte influencia del entorno que ofrece estos alimentos. Así, la publicidad, la información sobre nutrición, la disponibilidad de alimentos en el centro escolar, los precios y la educación juegan un papel fundamental en las elecciones de alimentos entre la población escolar.

En las escuelas, los Establecimientos de Consumo Escolar (ECE), tiendas o kioscos escolares son espacios que pueden brindar mejores opciones de alimentos y generar hábitos saludables dentro del contexto escolar, ya que, al ofrecer alimentos de mayor calidad nutrimental, disminuye el riesgo de que los escolares desarrollen sobrepeso u obesidad. En los últimos años en México, se han implementado las escuelas de tiempo completo, razón por la cual se ofrece a los escolares un servicio de alimentación que busca proveer alimentos y bebidas acordes con una alimentación correcta, y con el aporte de energía adecuado a su edad, su condición de vida y los nutrimentos necesarios para su desarrollo; con higiene y seguridad. ${ }^{6}$

\section{4- Servicios de salud}

Promover la salud en el ambiente escolar implica abordar los determinantes conductuales, sociales y ambientales del proceso salud-enfermedad, construyendo ambientes saludables e inclusivos. Un establecimiento de educación promotor de salud es el que ha integrado en su proyecto educativo el objetivo de reforzar constantemente las oportunidades de salud y bienestar para todos quienes allí estudian y trabajan. ${ }^{7}$ De acuerdo con la Organización Mundial de la Salud, se requieren servicios de salud locales y regionales que atiendan la salud y educación para la salud de niños y adolescentes, a través de la provisión de servicios directos a los estudiantes y en asociación con las escuelas, como los servicios que se anotan en la tabla 1.

Tabla 1: Servicios de salud locales y regionales de atención a la salud en escuelas

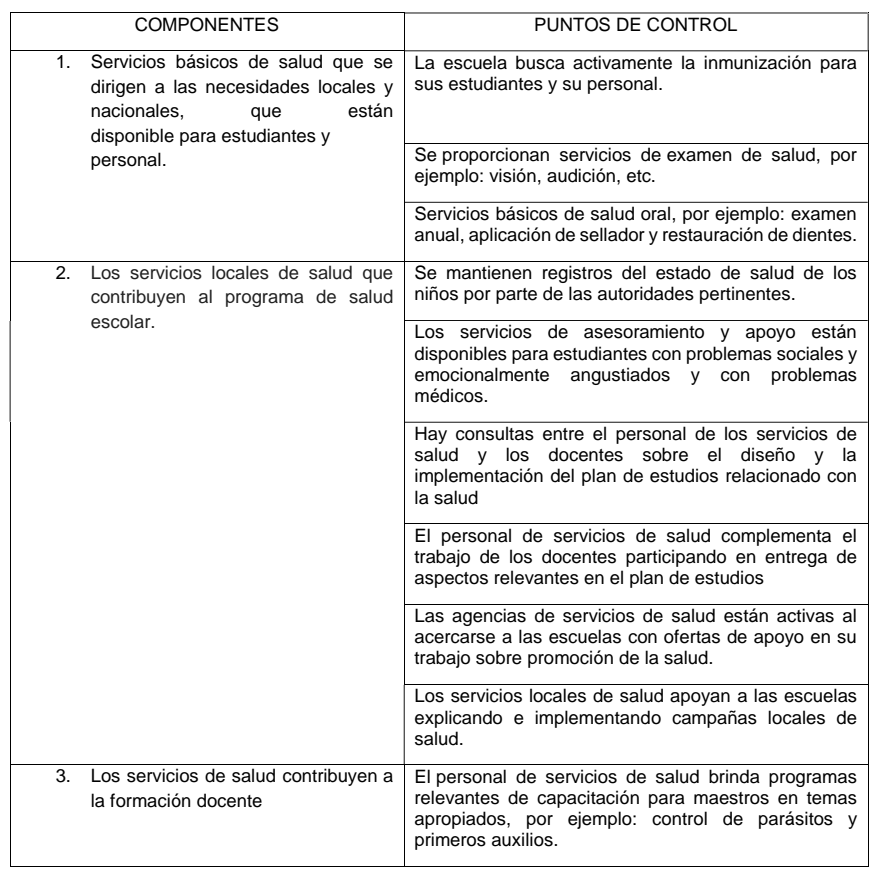

Fuente: World Health Organization. (1996). Regional guidelines: development of health-promoting schools-a framework for action. Manila: WHO Regional Office for the Western Pacific. ${ }^{8}$ 


\section{2- El entorno escolar como determinante de la salud y nutrición}

Una de las prioridades del mundo actual en términos educativos y de salud es la formación de buenos hábitos de alimentación desde la infancia, la evidencia científica muestra que las escuelas constituyen un entorno inmediato que apoya o impide el desarrollo de habilidades de los estudiantes para participar en comportamientos saludables.

\section{1- Clima psico-social}

El clima psicosocial, se puede separar en el clima escolar y el clima individual de la clase. El primero se puede delinear como expectativas para los estudiantes en cuanto a conducta y disciplina; y el último como una oportunidad de aprender a través de la autogestión del estudiante, y el reforzamiento de conductas positivas en el aula. ${ }^{9}$

La escuela es el espacio de socialización secundaria al hogar, en donde los escolares pasan un porcentaje importante de su tiempo en una etapa fundamental de su vida, por lo que es posible realizar allí acciones de socialización intencionada que les permitan adquirir las competencias o habilidades psicosociales básicas para ser y convivir. ${ }^{3}$

Uno de los problemas más relevantes en el entorno escolar es el "bullying" (acoso escolar o laboral) es un problema común. Sin embargo, es poco considerado como un diagnóstico en el campo de la salud asistencial. Es una forma de abuso que puede existir en cualquier ámbito donde convive un grupo de personas, pero ocurre con más frecuencia en el ámbito escolar de niños y adolescentes. Se presenta cuando una o más personas ejercen un comportamiento lesivo, intencional y recurrente contra otro $\mathrm{u}$ otros individuos, frecuentemente se caracteriza por un abuso sistemático del poder. Esta conducta puede expresarse de diferentes formas: como agresión física, verbal, psicológica y social. Sus consecuencias van más allá de la víctima; alcanzan al agresor y a los observadores. Las condiciones individuales y del ambiente determinan los factores de riesgo y los de protección para evitar su desarrollo. ${ }^{10}$

La escuela, como uno de los principales espacios de socialización del niño, tiene como misión orientar, formar y educar en valores y buenas costumbres; no obstante, puede llegar a propiciar la aparición de síntomas de problemas psicosociales. El entorno escolar es un determinante importante de la función psicosocial y también puede estar relacionado con la salud mental. ${ }^{11} \mathrm{El}$ entorno escolar como un determinante de la salud emocional puede marcar el presente y futuro de una persona, por ello es necesario abordarlo y atenderlo desde diferentes perspectivas, que involucren a la escuela y la familia.

\section{2- Infraestructura física}

Se conoce que el ambiente es un factor de cambio en las personas, por ello la infraestructura educativa es de gran importancia en las prácticas de salud y bienestar de los escolares. Se define a la infraestructura escolar como el conjunto de inmuebles, muebles, equipo, planes y programas de estudio, etc., que permite proporcionar oportuna y satisfactoriamente un servicio educativo.

Las condiciones de infraestructura de una escuela se pueden asociar frecuentemente con el nivel de rendimiento académico y la salud de los escolares debido a que estos pasan una gran parte del día dentro de las instituciones; sin embargo, la relación infraestructura escolar y estado nutricio no es tan clara, por lo que es necesario hacer estudios que contribuyan al análisis de esta relación ya que la escuela se percibe como el medio ideal para promover cambios de actitudes y valores en generaciones enteras, pero los temas pueden ser tan diversos que es necesario priorizarlos para tener generaciones más conscientes de los movimientos sociales, económicos, ecológicos y sobre todo generaciones preocupadas por el cuidado de su salud.

En el mismo sentido los patios, áreas verdes y zonas deportivas son fundamentales para el sano desarrollo y bienestar de los escolares, los primeros se refieren al lugar donde el menor puede obtener sano esparcimiento durante sus descansos e incluye la presencia de juegos infantiles como resbaladillas, columpios, pasamanos, entre otros; el segundo, se refiere al área delimitada donde se pueden ejecutar actividades físicas, por ejemplo canchas de futbol, basquetbol y volibol, gimnasios, entre otros. $^{3}$

un ambiente de aprendizaje se constituye por todos los elementos físico-sensoriales, como la luz, el color, el sonido, el espacio, el mobiliario, etc., que caracterizan el lugar donde un estudiante ha de realizar su aprendizaje, pues desde un punto de vista arquitectónico estos deben ser puntos a tomar en cuenta para posibilitar el aprendizaje, el confort, con el fin de ofrecerle al educando un ambiente acogedor, grato, atractivo, que le posibilite potenciar sus capacidades con base en sus intereses y necesidades. ${ }^{12}$

Los espacios escolares deben facilitar comportamientos saludables, como el acceso a bebederos de agua potable, caminos o veredas para que los niños y niñas caminen sin peligros, consuman sus alimentos en lugares limpios, y donde los mensajes en salud estén presentes.

\section{3- Seguridad escolar}

Un entorno saludable y seguro es aquel en el que los niños y jóvenes pueden desarrollarse plenamente, ofreciendo protección y desarrollo de capacidades para el cuidado de la salud. Para lograr entornos seguros y saludables en las escuelas, es necesario que la dirección escolar, los profesores, profesionales, concesionarios de 
establecimientos de consumo escolar, personal de apoyo, padres de familia y la comunidad educativa en general se integren activamente mediante el desarrollo de políticas escolares saludables, garantizando la existencia de entornos físicos adecuados y la creación de un ambiente de bienestar emocional y social positivo. ${ }^{6}$

Si compartimos el punto de vista de que la confianza es una condición indispensable para favorecer el aprendizaje de los alumnos y la construcción de relaciones de respeto y solidaridad en los miembros de la comunidad escolar, podremos comprender por qué el enfoque de convivencia representa una alternativa como modelo de gestión de la seguridad en las escuelas. Aprender a convivir en un ambiente de respeto y cuidado beneficia el aprendizaje y promueve el desarrollo de la autoestima, la valoración y el respeto que merecen las demás personas. ${ }^{13}$

En las últimas décadas se han incrementado considerablemente las investigaciones sobre violencia escolar. Esta violencia incluye varios tipos de conductas transgresoras que tienen lugar en escuelas e institutos, desde actos delictivos leves (como la rotura de cristales o las pintadas) hasta patrones de comportamiento más graves y relacionados con la agresión física y verbal a profesores y compañeros. Todas estas conductas impiden el normal desarrollo de la enseñanza y afectan gravemente al clima escolar y a las relaciones interpersonales de profesores y alumnos. En cuanto a las consecuencias negativas que la violencia entre compañeros tiene para las víctimas, ésta se ha relacionado con sentimientos de soledad y tristeza, baja autoestima y elevado ánimo depresivo. ${ }^{14}$

La seguridad en las escuelas es un asunto muy serio y de vital importancia no solo para los responsables del tema en cada plantel, sino para toda la sociedad en general ¿Qué sociedad puede funcionar adecuadamente sin contar con que sus hijas e hijos estén seguros? Seguramente ¡Ninguna!. ${ }^{15}$ Por lo que los profesores, padres de familia y autoridades locales, están llamados a colaborar como una unidad para garantizar en las escuelas y fuera de ellas, la seguridad de los niños y niñas.

\subsubsection{Seguridad alimentaria}

En la Cumbre mundial sobre alimentación en 1996 se definió a la seguridad alimentaria: Cuando toda persona en todo momento tiene acceso económico y físico a suficientes alimentos inocuos y nutritivos para satisfacer sus necesidades alimentarias y preferencias en cuanto alimentos a fin de llevar una vida sana y activa. En contraparte, una definición ampliamente aceptada de inseguridad alimentaria, es la siguiente: inseguridad alimentaria es la disponibilidad limitada o incierta de alimentos nutricionalmente adecuados e inocuos, o la capacidad limitada e incierta de adquirir alimentos adecuados en formas socialmente aceptables. La seguridad alimentaria, conjuntamente con la seguridad a tener buena salud, determinan la seguridad nutricional. ${ }^{3}$
En México, con base en los datos del 2008 , el $54.2 \%$ de la población se ubica con seguridad alimentaria, $24.2 \%$ presenta un grado de inseguridad alimentaria leve, $12.8 \%$ con inseguridad alimentaria moderado y un $8.8 \%$ con grado de inseguridad alimentaria severo. Es decir, que poco más de una quinta parte de la población mexicana $(21.6 \%)$ sufre carencias por acceso a la alimentación.

De acuerdo a los mismos datos, el estado de Hidalgo, se ubica como la sexta entidad del país con mayor porcentaje de población en pobreza multidimensional extrema y moderada, pues presenta mayor grado de inseguridad alimentaria que el promedio nacional: el $29 \%$ de la población estatal presenta un grado de inseguridad alimentaria leve, $15.2 \%$ con inseguridad alimentaria moderado y el $9.1 \%$ con grado de inseguridad alimentaria severo, mientras que el $46.7 \%$ de la población disfruta de seguridad alimentaria; lo que establece que, casi una cuarta parte de la población hidalguense $(24.3 \%)$ sufre carencias por acceso a la alimentación. Los escolares de Hidalgo se distribuyen por grados de inseguridad alimentaria de la siguiente manera: $29.6 \%$ inseguridad leve, $25.1 \%$ inseguridad moderada, $10.7 \%$ inseguridad severa.

Los niños que participan en experiencias educativas sobre seguridad alimentaria y nutrición tienen la oportunidad de aumentar su conciencia acerca de los comportamientos correctos para prevenir enfermedades transmitidas por los alimentos y mejorar su propio pensamiento crítico sobre el consumo de alimentos. ${ }^{16}$

La seguridad alimentaria determina no sólo el estado de nutrición y salud actual de la población infantil, sino que condiciona su calidad de vida en la edad adulta y la mala nutrición de las generaciones futuras.

\section{4- Transporte y movilidad escolar}

Se ha demostrado que existe una asociación entre el uso del transporte y la condición de sobrepeso u obesidad en escolares, en un estudio los niños y niñas que utilizaban transporte (bus de colegio, auto de familia, transporte público, o transporte privado), comparados con lo que caminaban a la escuela, se encontró un $75 \%$ más de riesgo para tener sobrepeso u obesidad. ${ }^{17}$

El sobrepeso y obesidad en los escolares ha ido en aumento, debido a los cambios en los estilos de vida, principalmente el sedentarismo motivado por el uso de transporte escolar, que además propicia un alto consumo de alimentos que aportan energía. ${ }^{18}$ Es necesario el desarrollo y la implementación de políticas enfocadas en la promoción y educación en el uso de medios de transporte alternativos al vehículo personal. Incentivar el uso del transporte público y de formas activas de movilidad (caminar o andar en bicicleta) lo que deberían ser prioridades en los planes de desarrollo urbano. Los niños que habitualmente caminan hacia la escuela son más activos que aquellos que se desplazan en auto. La actitud de los niños hacia formas alternativas de movilidad se relaciona con las opiniones y actitudes parentales. Específicamente, con la elección que los 
padres realizan sobre el medio de transporte en que sus hijos concurren a la escuela. La elección del automóvil se ve favorecida por factores tanto contextuales como psicológicos. Entre los factores contextuales se encuentran las distancias a recorrer, y la evaluación del ambiente en términos de seguridad y preferencia. Entre las variables psicológicas, el valor simbólico del automóvil, o la necesidad de cumplir expectativas personales y sociales tienen un peso importante.

Resulta relevante que durante la infancia se transmita que la bicicleta es un medio de transporte que tiene beneficios individuales, ambientales y sociales. Por ejemplo, a nivel de la salud individual, ir a la escuela en bicicleta ha sido asociado a la reducción del sedentarismo y a la disminución del índice de masa corporal y de la obesidad en niños. Con respecto al sedentarismo, existen estudios que plantean una relación positiva entre las tasas de obesidad y el uso del automóvil. Esto se observa claramente al comparar países de acuerdo con sus formas típicas de movilidad. Existen países, como es el caso de Suecia y Dinamarca, donde un porcentaje importante de los viajes urbanos se realiza en bicicleta o caminando, y los niveles de obesidad se mantienen bajos; a diferencia de otros como Brasil donde las tasas de obesidad han aumentado junto con el uso masivo del automóvil. ${ }^{19}$

\section{3- Normas que regulan la participación de la comunidad en la salud y nutrición escolar}

La Secretaría de Salud es el organismo encargado de emitir las Normas Oficiales Mexicanas que regulan muchos aspectos de nuestra vida que están relacionados con la salud de los escolares. ${ }^{20}$ Las Normas Oficiales Mexicanas (NOM) son de carácter obligatorio y son elaboradas por las dependencias del Gobierno Federal. En la tabla 2 se seleccionaron algunas normas que están relacionadas con el cuidado de la salud del escolar.

Tabla 2: Normas Oficiales Mexicanas para el cuidado del escolar.

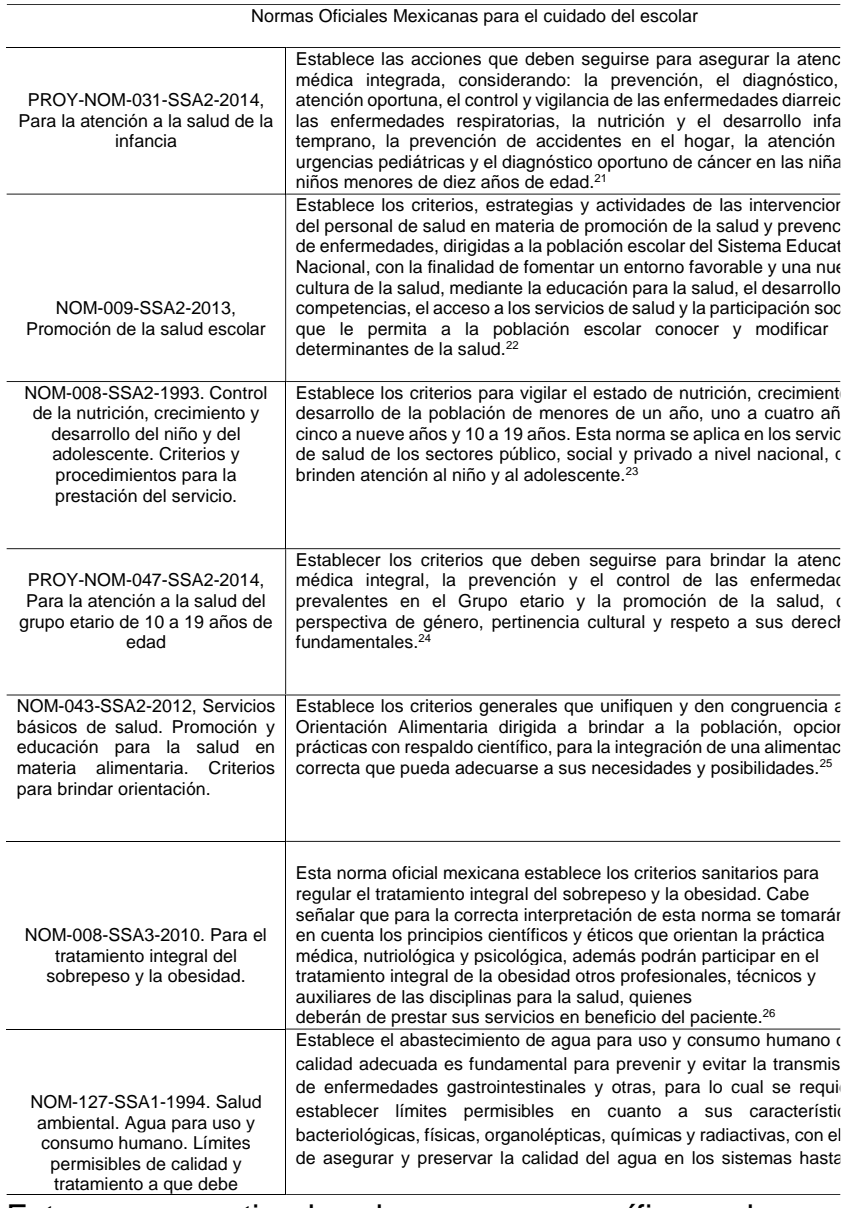

Estas normas atienden de manera específica cada uno de los aspectos de la vida del escolar, generan una atmósfera de seguridad, bienestar y calidad para garantizar un mejor desarrollo escolar y humano. Sin embargo, su cumplimiento depende de las entidades responsables, y generalmente están supeditadas a los recursos disponibles para su aplicación y cumplimiento.

\section{1- Consejo de salud escolar}

En la actual coyuntura de inseguridad que enfrenta nuestro país, las escuelas de educación básica siguen siendo uno de los espacios más seguros con que cuenta la sociedad, donde las niñas, los niños y los jóvenes crecen y se desarrollan física, emocional y socialmente. Para asegurar que así continúen, se ha propuesto reforzar la seguridad y la cultura de la prevención en las escuelas, con la colaboración activa y responsable de madres y padres de familia o tutores, así como del personal directivo, docente y demás miembros de la comunidad escolar.En este sentido, cobra especial relevancia la instalación y funcionamiento de los órganos formales de participación, como el Consejo Escolar de Participación Social (CEPS).

Los Consejos Escolares de Participación Social cumplen con el propósito de favorecer el bienestar escolar, promoviendo actividades y articulando esfuerzos para incidir en la mejora educativa y en el desarrollo integral de los alumnos. ${ }^{28}$ Los consejos escolares, pueden contribuir a disminuir la disponibilidad de alimentos y 
bebidas de bajo contenido de nutrientes y energía en las escuelas intermedias y secundarias. ${ }^{29}$

\section{2- Integración y operación del CEPS}

De acuerdo con el artículo $6^{\circ}$ del acuerdo 716 que establecen los lineamientos para la constitución, organización y funcionamiento de los consejos de participación social en la educación (CEPS), establece que, en cada escuela pública de educación básica, la autoridad escolar hará lo conducente para que se constituya y opere un CEPS de conformidad con el artículo 69 de la Ley General de la Educación.

De acuerdo con el segundo párrafo del Artículo 69 de la Ley General de Educación, el CEPS se integra con los padres y madres de familia y representantes de sus asociaciones, maestros y representantes de su organización sindical, directivos de la escuela, exalumnos, así como miembros de la comunidad interesados en el desarrollo de la propia escuela. Sus integrantes reciben el nombre de consejeros y se describe su integración en la tabla 3.

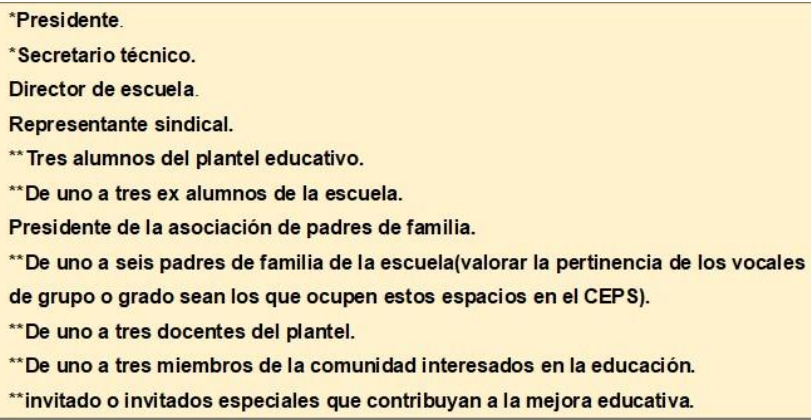

$\left({ }^{\star}\right)$ Elegidos en asambleas del conjunto de integrantes del conseio Escolar de Participación Social. Art. $4^{\circ}$.

Tabla 3: Integrantes de los Consejos Escolares de Participación Social (CEPS)

Para integrar un Consejo Escolar de Participación Social (CEPS) es importante considerar algunas acciones previas. Una de ellas se refiere a que en la comunidad escolar se realice un trabajo de sensibilización con todos los actores escolares, especialmente con las madres y los padres de familia o tutores, quienes deberán saber las ventajas y los beneficios que aporta el trabajo activo y coordinado del CEPS.

El director, con el apoyo de la Asociación de Padres de Familia (APF), puede organizar una sesión de información y análisis sobre la importancia de la participación social en la escuela, a través de los CEPS.

- A esta sesión informativa se debe invitar al presidente, al secretario, al tesorero y a los vocales de grupo de la APF, así como a representante(s) del colectivo docente. Pueden ser invitados otros actores de la comunidad, por ejemplo, representantes del gobierno municipal y ex alumnos, entre otros.
- En escuelas comunitarias e indígenas es importante contar con la presencia de las autoridades de la comunidad.

- La reunión que se organice debe tener bien definido lo siguiente:

- El tiempo de duración: es recomendable de 1 a 2 horas. - La sesión sólo debe tratar el tema de la participación social en la educación.

- Se debe enviar previamente a la reunión una orden del día, al final se destinará un tiempo determinado para asuntos generales, en donde se anotarán otros asuntos que indique la comunidad para que se atiendan posteriormente.

- Destinar un tiempo determinado para cada punto de la orden del día.

- Al final de cada punto debe formularse una conclusión en la que todos o la mayoría estén de acuerdo.

- Se sugiere que en la sesión se traten puntos como los siguientes:

- ¿En qué consiste la participación social en la escuela?, ¿por qué es importante?

- En la escuela, ¿cuál es el papel de los padres de familia o tutores y de otros actores externos en el marco de la participación social?

- ¿Cuál es la necesidad y la ventaja de formar un CEPS? - ¿Cuáles son las funciones del CEPS?, ¿cómo se coordinará con la APF y el Consejo Técnico Escolar para desarrollar sus funciones y actividades en pro de la mejora de la calidad educativa?

Es importante que los participantes en esta reunión puedan comentar sus percepciones y dudas 0 inquietudes y que éstas puedan ser atendidas para generar confianza y credibilidad y, con ello, crear mejores condiciones, a fin de integrar con responsabilidad el CEPS de la escuela.

Para integrar el CEPS es importante tomar en cuenta el nivel educativo, la modalidad y la matrícula escolar, pues dependiendo de ello podrán integrarse más o menos padres de familia, alumnos, ex alumnos y maestros. Lo que debe cuidarse es que el CEPS esté formado por $50 \%$ de padres de familia más uno.

Los miembros del Consejo Escolar durarán en su encargo un periodo de dos años, con la posibilidad de reelegirse por un periodo adicional. En caso de que algún miembro se separe del CEPS, puede ser sustituido temporalmente con otra persona, hasta que se celebre la siguiente asamblea de la comunidad escolar, donde se solicitará que se realice el nombramiento de un nuevo integrante del CEPS. Los cargos que desempeñen los consejeros son honoríficos. El Consejo Escolar, para desarrollar sus funciones, debe organizarse en comités a fin de ser un efectivo apoyo a la escuela. El Acuerdo 535 señala ocho comités; no obstante, pueden formarse tantos como requiera la escuela. 


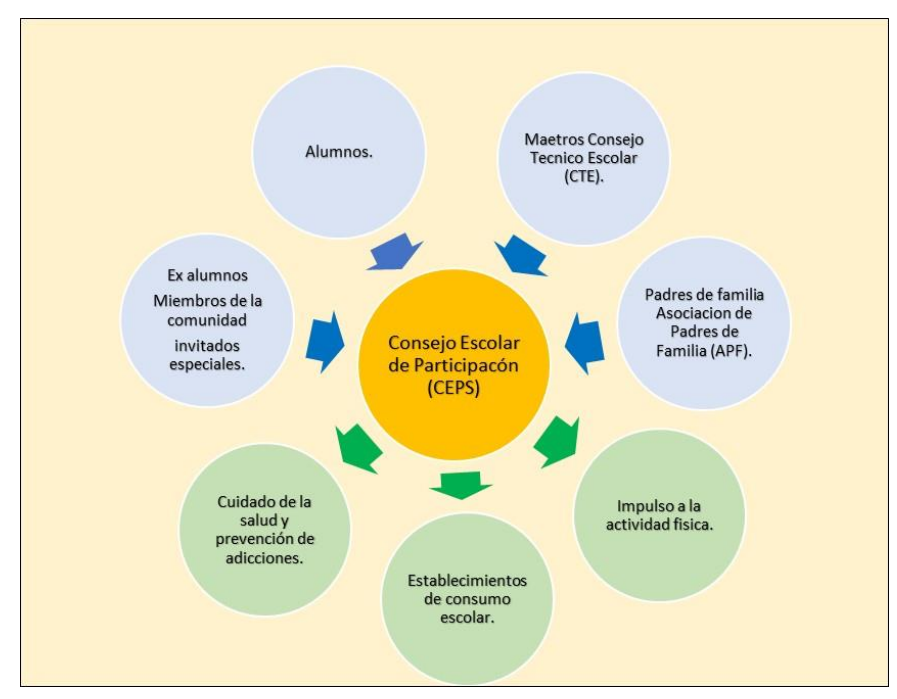

Figura 2: integración y participación del consejo escolar en la salud y nutrición.

Fuente: Elaborado con base en el acuerdo 716 que establecen los lineamientos para la constitución, organización y funcionamiento de los consejos de participación social en la educación y nutrición.

\subsection{Funcionamiento de los CEPS}

Conforme a los lineamientos generales para la operación de los Consejos Escolares de Participación Social, Capítulo I, Artículo 3, de las Disposiciones generales del Acuerdo 535, los CEPS deberán:

- Propiciar la colaboración de directivos, maestros, madres y padres de familia, así como de representantes de las asociaciones de estos últimos, para realizar convocatorias de trabajos específicos que permitan el mejoramiento de las instalaciones escolares, tomar nota de los resultados de las evaluaciones que realicen las autoridades educativas, conocer las metas educativas y apoyar actividades extraescolares.

- Informar a las madres y los padres de familia o tutores el monto de los recursos que, en su caso, sean otorgados a la escuela a través de programas federales, estatales o locales, los cuales serán ejercidos de acuerdo con la normatividad aplicable.

- Promover ante la Asociación de Padres de Familia o la agrupación equivalente, que ésta informe a la comunidad educativa el monto y uso del conjunto de los recursos que hubiera recabado.

- Promover que la comunidad educativa conozca el monto de los recursos que sean recabados por el CEPS y que provengan de cualquier fuente distinta a la mencionada en los dos párrafos anteriores.
- Tomar nota de los resultados de las evaluaciones que realicen las autoridades educativas, y exhortar al personal directivo y docente para que con base en los resultados se establezcan metas a fin de mejorarlos en la siguiente evaluación, haciendo del conocimiento de madres y padres de familia o tutores esta información.

- Respaldar las actividades cotidianas de la escuela, las cuales promoverán la integración, el conocimiento y los valores entre las familias y los miembros de la comunidad educativa, así como la gestión ante las autoridades educativas para la incorporación de la escuela a los programas federales: Escuelas de Tiempo Completo (PETC), Escuelas de Calidad (PEC), Escuela Segura (PES), Escuela Siempre Abierta (proesa), entre otros.

- Propiciar la colaboración entre padres de familia o tutores y sus asociaciones con el resto de la comunidad educativa para: organizar acciones que tengan por objeto incorporar a la escuela en los programas de lectura que existan; promover el uso y mejora de la biblioteca escolar y crear círculos de lectura; impulsar el mejoramiento de la infraestructura, la protección civil, la seguridad en las escuelas, el apoyo a la activación física, el desaliento de las prácticas que generen violencia entre pares, el consumo de alimentos saludables y el cuidado al medio ambiente; organizar actividades deportivas, recreativas, artísticas o culturales y, en general, desarrollar otras acciones en beneficio de la escuela.

- Abstenerse de intervenir en aspectos laborales, políticos o religiosos, de acuerdo con lo que señala el Artículo 73 de la Ley General de Educación.

\subsection{Comités que integran el Consejo de Participación Social y tipo de actividades que desarrollan}

Los CEPS conformarán comités por medio del acuerdo y la colaboración de la comunidad escolar para atender diversos asuntos y problemáticas escolares.

Es de vital importancia que los miembros del CEPS conozcan la planeación escolar o participen en su diseño, dependiendo de las condiciones de la escuela, con el propósito de acordar sus acciones para evitar duplicidad o empalme de actividades.

Si en la escuela ya se cuenta con una planeación, es conveniente que se dé a conocer al CEPS una vez integrado, para identificar las acciones que ya están programadas y diseñar otras que complementen 0 fortalezcan los mismos propósitos y metas establecidos. No ayuda a la organización escolar el diseño y la ejecución de planeaciones paralelas.

Las las acciones que el CEPS proyecte realizar deberán formar parte de la planeación general de la escuela. Si bien el Acuerdo 535 propone la creación de ocho comités, se sugieren, como ejemplo, algunos más, considerando las necesidades que pudieran existir en las escuelas. Es de gran importancia que el colectivo escolar valore la situación propia de la escuela para tomar decisiones, en la tabla 4 se anotan los comités que están relacionados con la salud y nutrición de los escolares. ${ }^{30}$ 
Tabla 4: Comités relacionados en salud y nutrición escolar establecidos en el Acuerdo 535.

\begin{tabular}{|c|c|}
\hline Comité & Actividades \\
\hline IV. Impulso a la activación física & $\begin{array}{l}\text { - Conocer las orientaciones emitidas por la SEP } \\
\text { para desarrollar la activación física. } \\
\text { - Identificar las condiciones de la comunidad } \\
\text { escolar y del plantel para la realización de } \\
\text { acciones dirigidas a la activación física. } \\
\text { - Convocar a toda la comunidad escolar para que } \\
\text { hagan sugerencias y para atender esta importante } \\
\text { actividad. } \\
\text { - Acordar con los maestros y directivos la forma en } \\
\text { que podrá desarrollarse esta actividad con la } \\
\text { participación de todos. No es tarea exclusiva de } \\
\text { los maestros de educación física - en caso de que } \\
\text { cuenten con ellos-, pero sí pueden proporcionar } \\
\text { orientación en cuanto a la manera en que puede } \\
\text { realizarse esta actividad con el apoyo de los } \\
\text { maestros en cada grupo y de los padres de familia } \\
\text { en casa. } \\
\text { - Registrar en la planeación del CEPS el acuerdo } \\
\text { para la realización de esta actividad, misma que } \\
\text { estará alineada e incluida en la planeación de la } \\
\text { escuela. } \\
\text { - Coordinar con la escuela e instituciones afines } \\
\text { acciones que promuevan la activación física. } \\
\text { - Dar seguimiento a las acciones realizadas y } \\
\text { valorar los beneficios obtenidos con este tipo de } \\
\text { actividades. } \\
\text { - Sugerir a los alumnos y padres de familia } \\
\text { acciones complementarias que favorezcan la } \\
\text { activación física fuera de la escuela. }\end{array}$ \\
\hline VII. Establecimientos de consumo escolar & $\begin{array}{l}\text { - Revisar los "Lineamientos generales para el } \\
\text { expendio o distribución de alimentos y bebidas en } \\
\text { los establecimientos de consumo escolar de los } \\
\text { planteles de educación básica". } \\
\text { - Analizar el "Reglamento de cooperativas } \\
\text { escolares" para considerar sus disposiciones y y } \\
\text { complementarlas con los "Lineamientos generales } \\
\text { para el expendio o distribución de alimentos y } \\
\text { bebidas en los establecimientos de consumo } \\
\text { escolar de los planteles de educación básica". } \\
\text { - Supervisar que el o los establecimientos de } \\
\text { consumo escolar se apeguen a los documentos } \\
\text { revisados anteriormente. } \\
\text { - Poner especial atención en el tipo de alimentos } \\
\text { que se consumen, identificar su valor nutricional, } \\
\text { tomando en cuenta su contexto. } \\
\text { - Informar y sensibilizar a la comunidad escolar } \\
\text { sobre la importancia del consumo de alimentos } \\
\text { saludables, si es posible, elaborar una gráfica del } \\
\text { tipo de alimentos que más se consumen y su valor } \\
\text { nutricional. } \\
\text { - Monitorear la tienda escolar para observar la } \\
\text { venta de alimentos que no cumplan con las } \\
\text { características establecidas por la Secretaría de } \\
\text { Salud. } \\
\text { - Impulsar el consumo de alimentos saludables al } \\
\text { interior de la escuela, alrededor de la misma y en } \\
\text { los hogares de los alumnos. }\end{array}$ \\
\hline
\end{tabular}

\section{4- Participación de la comunidad en la salud y nutrición escolar}

La oportunidad de sentarse todos juntos (padres, madres, profesorado, personal no docente, representantes del ayuntamiento, equipo directivo, etc. para coordinar y orientar sobre posibles planes e iniciativas o aspectos educativos relevantes, supone una conquista de la democracia en el campo educativo. El desarrollo democrático de la sociedad considera a la escuela como una empresa de todos, aunque en diferentes niveles, cada uno tiene su papel muy bien definido. La educación debe ser meditada y elaborada con la implicación de toda la sociedad: padres y madres, alumnado, docentes, etc.

Todos sabemos que la escuela no es el único agente que educa y sabemos que el papel que desarrollan los diferentes agentes socializadores, es por ello, que se debe facilitar los cauces para que toda la comunidad educativa pueda asumir su responsabilidad de participación.

Las dos instituciones familia y escuela deben trabajar en consonancia para favorecer el pleno desarrollo del alumnado en sus diferentes ámbitos de la personalidad (cognitivo, social, afectivo, motor y moral), ambas instituciones están interrelacionadas y una sin la otra estarían incompleta. Si los padres y madres delegan en la escuela o no trabajan de forma conjunta, la labor de esta es sumamente complicada, ya que tendrían que ocuparse de aspectos de socialización primaria. Por ello, la importancia de la complementariedad entre padres y madres y escuela es vital.

Para que exista una autentica participación, el centro educativo debe facilitar a la familia la planificación y toma de decisiones con respecto a la educación de sus hijos o hijas y no asumir aquello que determine la escuela. Es necesario una búsqueda de objetivos comunes, con motivaciones afines, la participación de los padres y las madres debe ser gradual y sistemática, aunando esfuerzos comunes con el centro y el profesorado. ${ }^{31}$

\subsection{Problemas de los padres de familia}

Los padres de familia, por su parte, también nombran la falta de tiempo y su desinterés en las actividades escolares para explicar porque la participación social no se ha consolidado. Para los directores y supervisores, es la excesiva carga de trabajo, desarticulación y operación simultánea de numerosos programas escolares, falta de recursos y el reducido margen de decisión que tienen las escuelas lo que entorpece la participación social.

Un primer paso para motivar la participación de los padres de familia es hacer de su conocimiento cómo hacerlo, y por otra parte las autoridades escolares deben involucrar a los padres para fortalecer las acciones de la escuela y no verlo como una forma de fiscalización de los recursos de la escuela. ${ }^{32}$

\subsection{Participación de los padres en la nutrición escolar}

Se ha identificado que los padres de familia no se involucran en el mejoramiento de la salud y nutrición de los escolares, porque tienen desconocimiento de lo que deben hacer frente a diversas situaciones y porque no se sienten capaces de jugar un papel orientador para sus hijos. En un estudio realizado en escuelas urbanas, sólo el $5 \%$ de los padres de familia se percibió como capaz de incluir verdura, fruta y agua en el refrigerio escolar, y la mayoría no consideró importante la realización de actividad física en sus hijos; en tanto que el $80 \%$ de los profesores percibió que existe un problema de obesidad y sobrepeso en sus estudiantes, y que esta condición se debe al descuido de los padres al permitir que sus hijos consuman una gran cantidad de alimentos industrializados. Esta paradoja, donde por un lado los padres de familia no se asumen como responsable de la salud de sus hijos, y los profesores perciben que existe un grave problema de obesidad, nos indica que no existe comunicación, entre los actores del contexto escolar, que son determinantes para realizar acciones que mejoren la alimentación de los niños y niñas involucrados. Si bien el fomento tiene que dirigirse principalmente a los escolares, también tiene que involucrar a todos los actores sociales que son factores del cambio, como los 
padres, la familia, las instituciones educativas, las autoridades de la comunidad y, muy especialmente, la industria productora de alimentos procesados y bebidas, así como a la industria de la publicidad impresa y electrónica. ${ }^{33}$

\section{Conclusiones}

Actualmente operan diferentes programas de atención a la salud y nutrición dirigidos a los niños y niñas que asisten a la escuela, los programas forman parte indiscutible del proceso educativo cuya función es formar de manera integral a los alumnos. Se trata tanto de transmitir conocimientos como de favorecer la adquisición de habilidades que ayuden a afrontar la realidad del modo más eficaz y, para lograr este objetivo, se requiere de la participación y el compromiso de los profesores, padres de familia, autoridades educativas y autoridad local.

Cómo se ha descrito uno de los elementos que más determina la salud y bienestar de los escolares, es la formación de buenos hábitos de alimentación desde la infancia por lo que las escuelas constituyen un entorno inmediato que apoya o impide el desarrollo de habilidades de los estudiantes para participar en comportamientos saludables. Una educación de calidad, junto a la realización de actividades multidisciplinarias, son instrumentos que pueden contribuir a reducir las desigualdades en salud.

En México existen normas que regulan la participación de la comunidad en la salud y nutrición escolar. Las Normas Oficiales Mexicanas establecen los criterios, estrategias, actividades y procedimientos aplicables al Sistema Nacional de Salud en todas las unidades que proporcionan atención a los niños residentes en la República Mexicana. Sin embargo, la mala comunicación con los padres desde las escuelas y la falta de involucramiento de los padres no favorece la construcción de una sociedad colaborativa en la toma de decisiones y desarrollo de acciones con corresponsabilidad, transparencia y rendición de cuentas, para favorecer la salud del escolar.

La escuela y la comunidad deben de trabajar en consonancia para favorecer el pleno desarrollo del escolar, ambas instituciones están interrelacionadas y una sin la otra estaría incompleta. Para mejorar la participación de la comunidad es necesario considerar a cada integrante de la comunidad como un ser distinto y, a la vez, con los mismos derechos y responsabilidades para opinar, sugerir u observar situaciones educativas que impacten favorablemente en el estado de salud de los escolares.

La escuela es un ambiente idóneo para desarrollar una cultura democrática, al poner en práctica, de manera organizada, las capacidades y los esfuerzos de todos sus integrantes, a fin de contribuir al logro de los propósitos educativos y de la formación integral de sus estudiantes.

\section{Bibliografía:}

1. Macias M Adriana Ivette, Gordillo S Lucero Guadalupe, Camacho R Esteban Jaime. Hábitos alimentarios de niños en edad escolar y el papel de la educación para la salud. Rev. chil. nutr. [Internet]. 2012 Sep [citado 2017 Dic 06] ; 39( 3 ): 4043 Disponible en: http://www.scielo.cl/scielo.php?script=sci_arttext\&pid=S071775182012000300006\&lng=es. http://dx.doi.org/10.4067/S071 7-75182012000300006

2. Lohrmann, D. K. A Complementary Ecological Model of the Coordinated School Health Program. Public Health Reports, (2008). 123(6), 695-703.

3. Galván, M., \& Rodríguez, G. L. Perfil nutricional de escolares de Hidalgo 2010: Estado de nutrición de escolares y variables del contexto familiar, escolar e individual. Educación y Salud Boletín Científico de Ciencias de la Salud del ICSA, (2013). 1(2).

4. Mujica Johnson, Felipe Nicolás, Orellana Arduiz, Nelly del Carmen, Aránguiz Aburto, Hugo Augusto, \& González Fuenzalida, Héctor Ismael. Atribución emocional de escolares de sexto año básico en la asignatura de Educación Física y Salud. Educación Física y Ciencia, (2016). 18(2), 00. Recuperado en 07 de diciembre de 2017, de http://www.scielo.org.ar/scielo.php?script=sci_arttext\&pid=S2 314-25612016000200007\&lng=es\&tlng=es.

5. Alvis-Chirinos, Katherineet al. Medición de la actividad física mediante acelerómetros triaxiales en escolares de tres ciudades del Perú. Revista Peruana de Medicina Experimental y Salud Pública [online]. 2017, v. 34, n. 1 [Accedido 7 Deciembre 2017] pp. 28-35. Disponible en: <https://doi.org/10.17843/rpmesp.2017.341.2764>. ISSN 17264642. https://doi.org/10.17843/rpmesp.2017.341.2764.

6. Galván, M., \& Rodríguez, G. L. Modelo Operativo de Establecimientos de Consumo Escolar Saludable (ECSAL). Prevención de Sobrepeso y Obesidad en escolares PESOEH, 2018, Educación y Salud Boletín Científico de Ciencias de la Salud del ICSA. Disponible en https://www.researchgate.net/publication/323227657_Modelo_ Operativo_de_Establecimientos_de_Consumo_Escolar_Saluda ble_ECSALPrevencion_de_Sobrepeso_y_Obesidad_en_escola res_PESOEH [accessed Feb 28 2018].

7. Salinas C Judith, Vio del R Fernando. PROGRAMAS DE SALUD Y NUTRICIÓN SIN POLÍTICA DE ESTADO: EL CASO DE LA PROMOCIÓN DE SALUD ESCOLAR EN CHILE. Rev. chil. nutr. [Internet]. 2011 Jun [citado 2017 Dic 08] ; 38( 2 ): 100-116. Disponible en: http://www.scielo.cl/scielo.php?script=sci_arttext\&pid=S071775182011000200001\&lng=es. http://dx.doi.org/10.4067/S071 7-75182011000200001

8. World Health Organization. Regional guidelines: development of health-promoting schools-a framework for action. Manila: WHO Regional Office for the Western Pacific. (1996).

9. Lohrmann, D. K. A Complementary Ecological Model of the Coordinated School Health Program. Public Health Reports, (2008). 123(6), 695-703.

10. Loredo-Abdalá, A., Perea-Martínez, A., \& López-Navarrete, G. E. Bullying": acoso escolar. La violencia entre iguales. Problemática real en adolescentes. Acta pediátrica de México, (2008). 29(4), 210-214.

11. Castro M Bibiana Andrea, Gaviria L Marta Beatriz. Clima escolar y comportamientos psicosociales en niños. Rev. Fac. Nac. Salud Pública [Internet]. 2005 July [cited 2018 Mar 01] ; 23( 2 ) : 59-69. Available from: http://www.scielo.org.co/scielo.php?script=sci_arttext\&pid=S0 120-386X2005000200007\&lng=en. 
12. García-Chato, G. I. Ambiente de aprendizaje: Su significado en educación preescolar. Revista de Educación y Desarrollo, (2014). 29.

13. Fierro Evans, María Cecilia. Convivencia inclusiva y democrática: Una perspectiva para gestionar la seguridad escolar. Sinéctica, (2013). (40), 01-18. Recuperado en 11 de diciembre de 2017, de http://www.scielo.org.mx/scielo.php?script=sci_arttext\&pid=S $1665-109 X 2013000100005 \& \operatorname{lng}=$ es\&tlng=en .

14. Cava, M. J., Buelga, S., Musitu, G., \& Murgui, S. Violencia escolar entre adolescentes y sus implicaciones en el ajuste psicosocial: un estudio longitudinal. Revista de psicodidáctica, (2010). 15(1).

15. Gobierno de México, Guía de intervención: Educación general secundaria. Jalisco, (2018). http://edu.jalisco.gob.mx/educaciongeneralsecundaria/sites/edu .jalisco.gob.mx.educaciongeneralsecundaria/files/guia_de_inter vencion_escolar_ecyv2.pdf

16. Traversa, A., Adriano, D., Bellio, A., Bianchi, D. M., Gallina, S., Ippolito, C., ... \& Decastelli, L. Food safety and sustainable nutrition workshops: educational experiences for primary school children in Turin, Italy. Italian journal of food safety, (2017). 6(1).

17. Roca Leslye, Mejía Salas Héctor. Prevalencia y factores de riesgo asociados a obesidad y sobrepeso. Rev. bol. ped. [Internet]. 2008 Ene [citado 2018 Ene 01] ; 47( 1 ): 812. Disponible en: http://www.scielo.org.bo/scielo.php?script=sci_arttext\&pid=S1 024-06752008000100003\&lng=es.

18. Oleas G Mariana. Prevalencia y factores de riesgo de sobrepeso y obesidad en escolares de la provincia de Imbabura: Ecuador. 2010. Rev. chil. nutr. [Internet]. 2014 Mar [citado 2018 Ene 02] ; 41( 1 ): 61-66. Disponible en: http://www.scielo.cl/scielo.php?script=sci_arttext\&pid=S071775182014000100008\&lng=es. http://dx.doi.org/10.4067/S071 7-75182014000100008.

19. Poó, Fernando Martín, López, Soledad Susana, Tosi, Jeremías, Nucciarone, María Isabel, \& Ledesma, Rubén Daniel. Educación vial y movilidad en la Infancia. Psicologia Escolar e Educacional, (2015). 19(2), 395. https://dx.doi.org/10.1590/2175-3539/2015/0192881

20. SEP. Estrategia 5 pasos para salud escolar. Mexico: Gob. mx. (2013). https://sep.gob.mx/work/appsite/basica/estrategia_5_pa sos.pdf

21. Secretaria de gobernación, PROY-NOM-031-SSA2-2014, Para la atención a la salud de la infancia,México: Diario oficial de la federación, 2014.

22. Secretaria de gobernación, NORMA Oficial Mexicana NOM009-SSA2-2013, Promoción de la salud escolar. México: Diario oficial de la federación. (2013). pp.http://www.dof.gob.mx/nota_detalle.php?codigo $=5324923$ \&fecha $=09 / 12 / 2013$.

23. Secretaria de gobernación, NOM-008-SSA2-1993. Control de la nutrición, crecimiento y desarrollo del niño y del adolescente. Criterios y procedimientos para la prestación del servicio. Mexico, Diario oficial de la federación, 1993.

24. Secretaria de gobernación, PROY-NOM-047-SSA2-2014, Para la atención a la salud del grupo etario de 10 a 19 años de edad, Mexico, Diario oficial de la federación, 2014.

25. Secretaria de gobernación, NOM-043-SSA2-2012, Servicios básicos de salud. Promoción y educación para la salud en materia alimentaria. Criterios para brindar orientación. Mexico, Diario oficial de la federación, 2012.
26. Secretaria de gobernación, NOM-008-SSA3-2010. Para el tratamiento integral del sobrepeso y la obesidad. Mexico, Diario oficial de la federación, 2010.

27. Secretaria de gobernación, NOM-127-SSA1-1994. Salud ambiental. Agua para uso y consumo humano. Límites permisibles de calidad y tratamiento a que debe someterse el agua para su potabilización. Mexico, Diario oficial de la federación, 1994.

28. Secretaria de educación pública, Manual de Seguridad Escolar Recomendaciones para protegernos de la inseguridad y la violencia, México, 2012. https://www.google.com.mx/url?sa=t\&rct=j\&q=\&esrc=s\&sour $\mathrm{ce}=$ web $\& \mathrm{~cd}=1 \& \mathrm{cad}=$ rja\&uact $=8 \& \mathrm{ved}=0$ ahUKEwjkua2rbjYAhXJYiYKHYgbC68QFggrMAA\&url=http\%3A\%2 F\%2Fwww.hgo.sep.gob.mx\%2Fcontent $\% 2 F p d f \% 2$ Fescsegura $\% 2$ FManual $\% 2520$ de $\% 2520$ Seguridad.pdf\&usg=AOvVaw0zB ErQqiPjNXHu1SSqdASK

29. Kubik, M. Y., Farbakhsh, K., \& Lytle, L. A. Two years later: wellness councils and healthier vending in a cohort of middle and high schools. Journal of Adolescent Health, (2011). 49(5), 550-552.

30. Secretaria de educación pública, ACUERDO número 716 por el que se establecen los lineamientos para la constitución, organización y funcionamiento de los Consejos de Participación Social en la Educación, México, 2013.

31. Carmen Jurado Gomez, la familia y su participación en la comunidad educativa. Innovación y experiencias educativas, revista digital, 2009. N.23.

32. Flores Crespo P. Ramírez Ramón A. La Participación Social en la Escuela en México. Una Revisión de Literatura, REICE, México, 2015, 13(3), 77-94.

33. Galván, Marcos \& López-Rodríguez, Guadalupe. Estrategia NUTREH, evaluación de la estrategia de atención a la nutrición infantil en municipios prioritarios del estado de Hidalgo (2016). 